\title{
Predicting bruise susceptibility in apples using Vis/SWNIR technique combined with ensemble learning
}

\author{
Yao Jian, Guan Jiyu, Zhu Qibing* \\ (Key Laboratory of Advanced Process Control for Light Industry (Ministry of Education), Jiangnan University, Wuxi 214122, China)
}

\begin{abstract}
Bruise susceptibility in fruits is an important indicator in evaluating risk factors for bruising caused by external factors. Prediction of the bruising susceptibility of fruit can provide useful information for proper postharvest handling and storage operations. In this study, visible and shortwave near-infrared (Vis/SWNIR) technique was used to develop nondestructive method for predicting the bruise susceptibility of apples. Vis/SWNIR spectra covering 400-1100 nm were collected for 300 'Golden Delicious' apples over a time period of three weeks after harvest. A pendulum-like device was used to simulate impact bruise at three impact energy levels of $1.11 \mathrm{~J}, 0.66 \mathrm{~J}$ and $0.33 \mathrm{~J}$. Bruise volumes were estimated from the digital images of the bruised apples by using the bruise thickness model. Three prediction models, i.e. partial least squares model (PLS), partial least squares model combined with successful projection algorithm (SPA-PLS), and selective ensemble learning based on feature selection (SELFS), for bruise susceptibility were developed for each impact energy level as well as for the pooled data. Compared with PLS and SPA-PLS model, SELFS gave the better prediction results for bruise susceptibility, with the correlation coefficient of $R_{p}=0.800-0.886$ for the prediction set, the root-mean-square error of 38.7$62.1 \mathrm{~mm}^{3 /} \mathrm{J}$ for the prediction set (RMSEP), and the residual predictive deviation $(R P D)$ of 1.78-2.14 for three impact energy level. For three impact energy levels, the RMSEP and RPD value obtained by SELFS model improved by $14.8 \%-20.0 \%$ and 15.0\%-24.5\% compared to PLS model, and 11.4\%-21.2\% and 11.5\%-27.1\% compared to SPA-PLS model, respectively. The SELFS model achieved relatively lower prediction accuracies for the pooled data, with the $R_{p}$ values of $0.731, R M S E P$ of $85.46 \mathrm{~mm}^{3} / \mathrm{J}$, and $R P D$ of 1.46 , which were also better than that of PLS model and SPA-PLS model. This research demonstrated that Vis/SWNIR technique combined with ensemble learning is promising technique for rapid assessment of bruise susceptibility of fruit, which would be useful for postharvest handling of fruit.
\end{abstract}

Keywords: apple, nondestructive detection, bruise susceptibility, visible/short-wave near-infrared technique, ensemble learning DOI: $10.25165 /$ j.ijabe. 20171005.2888

Citation: Yao J, Guan J Y, Zhu Q B. Predicting bruise susceptibility in apples using Vis/SWNIR technique combined with ensemble learning. Int J Agric \& Biol Eng, 2017; 10(5): 144-153.

\section{Introduction}

Bruising is the most common type of mechanical damage affecting fresh apples, which results from

Received date: 2016-09-29 Accepted date: 2017-06-11

Biographies: Yao Jian, PhD candidate, research interests: sensors and detecting technology, Email: Yaojian@jiangnan.edu.cn; Guan Jiyu, Master, research interest: nondestructive detecting technology of agricultural products; Email: Guanguanjiyu@ 163.com.

* Corresponding author: Zhu Qibing, PhD, Professor, research interests: nondestructive detecting technology and agricultural informatization. Key Laboratory of Advanced Process Control for Light Industry (Ministry of Education), Jiangnan University, Wuxi 214122, China. Email: Zhuqib@163.com. excessive impact and compression during harvest and transport and at all stages of postharvest handling operations ${ }^{[1]}$. Bruising degrades the quality and marketability of apples; even minor bruises may influence consumer acceptance, resulting in significant economic $\operatorname{losses}^{[2]}$. Extensive studies have been reported on bruise detection of apples, but these studies are mostly concentrated on the detecting bruise which has been produced during harvest, transport and postharvest handling operations ${ }^{[3]}$. Although detection of bruise can reduce the risk of delivering damaged fruit to the consumer market, it cannot provide useful information to reduce or prevent the incidences of bruising damage. Therefore, if some preventive measures were taken to 
understand and predict the bruise susceptibility and the factors affecting it, it would be useful to reduce or minimize incidences of bruising damage by taking proper actions $^{[4,5]}$. There are many factors affecting fruit bruising damage; these factors include inherent factors, such as fruit maturity, firmness and mass, and tissue water potential ${ }^{[6]}$. In addition, the amount of energy absorbed by fruit during impact is the other major bruise inducing factor ${ }^{[7]}$.

The susceptibility of fruit to bruising during impact is often defined as the amount or volume of damage resulting from unit energy absorbed, expressed in the units of $\mathrm{mm}^{3} / \mathrm{J}^{[8]}$. The value of bruise susceptibility reflects the ability of fruit resisting bruise damage caused by external forces. Predicting and quantifying bruise susceptibility can provide useful information or recommendations for proper fruit handling and storage $^{[9,10]}$. Researchers investigated the influence of impact factors and fruit properties on bruise susceptibility. Khan and Vincent ${ }^{[11,12]}$ reported that fruit texture is a major factor affecting bruise susceptibility in apples. Garcia et al. ${ }^{[13]}$ considered that fruit firmness is a factor influencing bruise susceptibility in apples. To evaluate bruise susceptibility in fruits, several measurement methods have been proposed. For example, Schoorl and Holl $^{[14]}$ measured the bruising resistance (expressed in energy per unit bruise volume) by dropping each apple onto a solid surface from a certain height. Opara et al. ${ }^{[15]}$ designed a pendulum-like device to evaluate bruise susceptibility in fruits. All these reported methods are destructive and, thus, can only be used for a small number of samples. There are considerable differences in bruise susceptibility among the fruit that are grown, harvested or storage under the same environment condition, due to the inherent biological variability. Therefore, it is desirable to evaluate bruise susceptibility of individual fruit.

In recent years, visible and shortwave near-infrared (Vis/SWNIR) spectroscopy has been extensively studied for assessing quality of fruits because of the advantages of rapid and nondestructive detection. More importantly, it can provide a large amount of information related to chemical and physical properties of fruits, which is helpful to achieve measurement of multiple quality parameters. Pan et al. ${ }^{[16]}$ used Vis/SWNIR spectra at 400-1100 nm to predict the soluble solid content of beet. Subedi and Walsh ${ }^{[17]}$ developed the prediction models for dry matter and total soluble solids content of banana and mango fruit using Vis/SWNIR with spectral region of 500-1050 $\mathrm{nm}$. Wu and Wang ${ }^{[18]}$ used Vis/SWNIR covering $600-1600 \mathrm{~nm}$ to evaluate the effects of simulated transport vibration levels on damage of tomato fruit. Given that the physical and chemical properties are main factors affecting bruise susceptibility of fruits, and they can be obtained by using Vis/SWNIR spectroscopy, hence, Vis/SWNIR spectroscopy is introduced in this work to detect bruise susceptibility. The specific objectives are to:

1) Measure the reflectance spectra of 'Gold Delicious' apples with different bruise susceptibility over 400-1100 nm;

2) Develop quantitative prediction models for apple bruise susceptibility using Vis/SWNIR spectra combined with selective ensemble learning algorithm.

\section{Materials and methods}

\subsection{Fruit samples}

A total of 300 fresh and bruise-free 'Golden delicious' apples with equatorial diameter between $70 \mathrm{~mm}$ and $80 \mathrm{~mm}$ were sampled from the Agricultural Experiment Station of Michigan State University in October 2013; the apples were stored at room temperature $\left(20^{\circ} \mathrm{C}-22^{\circ} \mathrm{C}\right)$ for one week prior the experiment. To develop a more robust and reliable prediction model, it is desirable to have apple samples with a wider range of distribution in the bruise susceptibility. It is well known that the fruit firmness is changed with the storage time, and finally resulting to the change of bruise susceptibility. Hence, tests were carried out with a time span over three weeks. Samples in amount of 60,120 and 120 were measured after one-week, two-week, and three-week storage time, respectively. Vis/SWNIR spectra were first acquired from the intact apples, and they were then subjected to impact damage tests to measure the bruise susceptibility. Three impact energy levels were used in this study to achieve different levels of bruise. For each week test, the samples were equally divided into three 
groups; apples for each group were subjected to impact test at one of the three energy levels. Hence, there were 100 samples for each impact energy level.

\subsection{Acquisition and processing of Vis/SWNIR spectra}

A spectrometer (LOE-USB, tec5USA INC., Plainview, NY, USA) covering the spectral region of 400-1100 nm was used for reflectance spectra acquisition in an interactance mode (Figure 1a). The apple was illuminated with a broadband light delivered from ring light guide of $25 \mathrm{~mm}$ in diameter. In the center of the probe, a light detector covering an area of $11 \mathrm{~mm}$ diameter was used to receive the light that had traveled through the flesh tissue. A minimum separating distance of $3.5 \mathrm{~mm}$ between the light illuminating ring and the detector ensured that only the light that has traveled through the apple tissue would be received by the probe (Figure 1b). A soft rubber sealing ring was used between the illuminating ring and the detector to block illuminating light from entering the detecting area directly. A sponge ring (5 $\mathrm{mm}$ thick) was attached to the periphery of the probe to block ambient light. The lamp power supply was set to $100 \mathrm{~W}$ and the integration time was set to $575 \mathrm{~ms}$. Considering the equatorial area of fruit is representative of the whole fruit and more homogeneous, compared to the other regions of the fruit, the equatorial area was chosen for acquiring Vis/SWNIR spectra. Two scans were acquired from the same location of each apple sample.

To compensate for the light source variation effect, the dark current and the reference current of white Teflon were acquired before and after spectra acquisition of each batch of apples. They were used for spectra correction based on the following equation

$$
I_{r}=\left(I_{s}-I_{d}\right) /\left(I_{t}-I_{d}\right)
$$

where, $I_{r}$ is the relative spectra; $I_{s}$ is the mean value of two spectra obtained from each apple sample; $I_{d}$ is the mean value of dark current; $I_{t}$ is the mean value of the reference spectra of white Teflon. All further analyses were carried out on the relative spectra.

Figure 2 shows the relative reflectance spectra of 300 'Golden delicious' apples for the vis/SWNIR regions. The dramatic decreasing in the relative reflectance spectra around $680 \mathrm{~nm}$ and $960 \mathrm{~nm}$ were observed. A downward peak around $680 \mathrm{~nm}$ reflects the maturity of apples accompanied with the change of fruit color caused by chlorophyll contents. The absorption regions in the short wave NIR at $840 \mathrm{~nm}$ and $960 \mathrm{~nm}$ were likely attributed to the sugar absorption bands and the combination effect of $\mathrm{OH}$ groups from carbohydrates and water. These features are related to the complex physical and chemical properties of the fruit; therefore, the spectra of fruit tissues can be used to evaluate fruit properties.

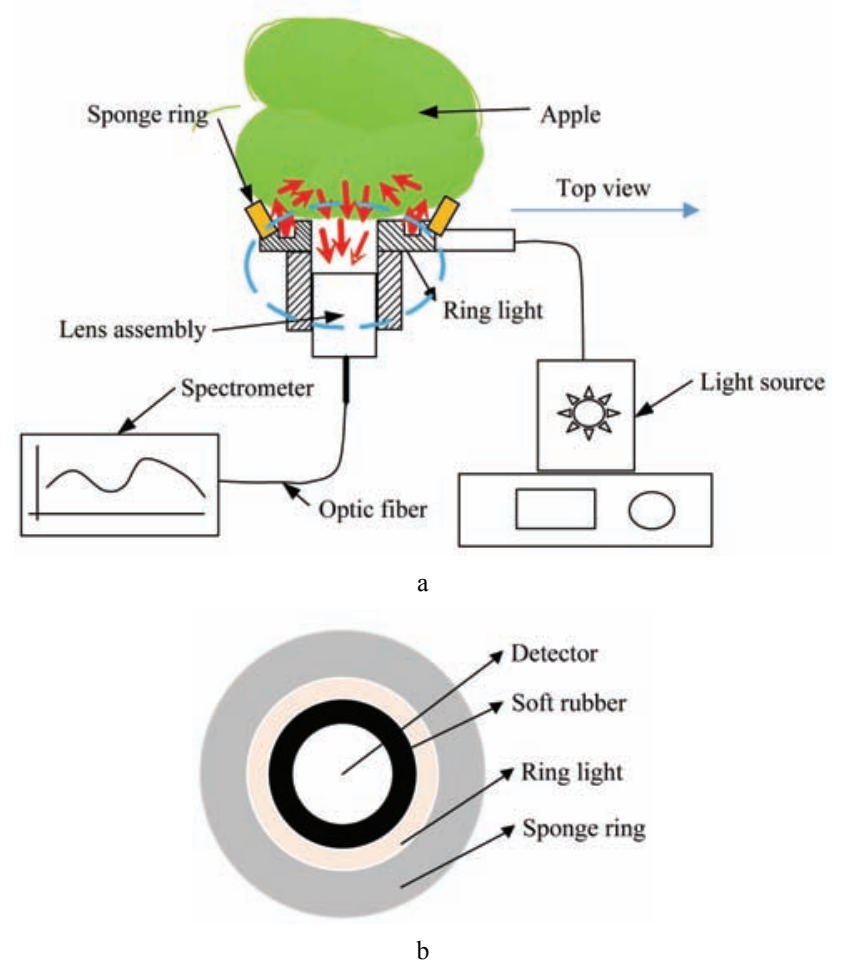

Figure 1 Schematic of the visible and near-infrared spectral acquisition system (a), the configuration of the lighting/detection probe (b)

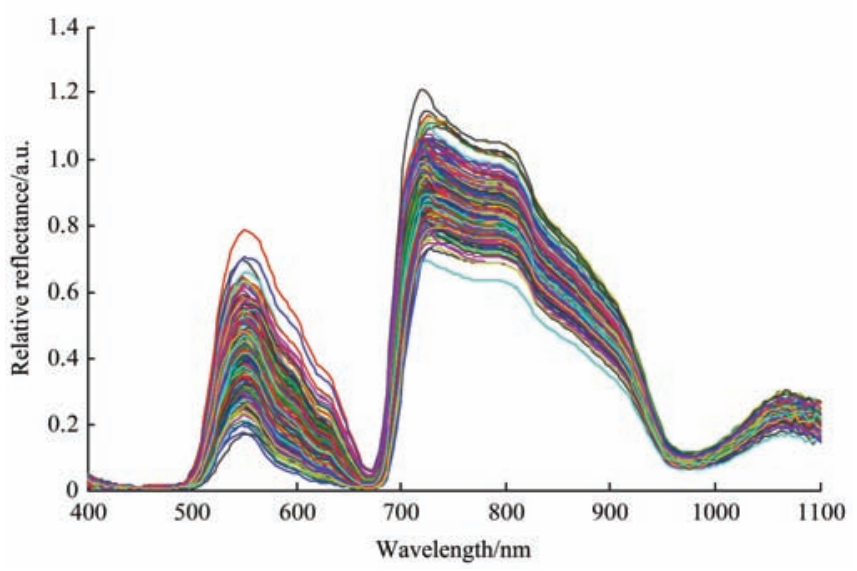

Figure 2 Relative reflectance spectra of 300 'Golden Delicious' apples at $400-1100 \mathrm{~nm}$

\subsection{Destructive instrumental test}

After collection of spectra, impact bruising tests were 
conducted by using a pendulum-like device. The impact bruising of apples in reality is caused by soft impacts from interactions between apples, belts, and etc. The wood ball is not as stiff as a steel ball, which can better mimic the bruised damage in transportation and postharvest handling operations. Hence, the wooden ball with diameter of $78 \mathrm{~mm}$ and $164.8 \mathrm{~g}$ of weight was used to introduce impact bruising of apples. Experiment showed that the used wooden ball can form a larger bruised surface on apple and reduce the calculation error produced by image segmentation. The wooden ball was mounted onto an aluminum rod which was approximately $157.2 \mathrm{~g}$ in weight and $483 \mathrm{~mm}$ in length. The other end of the rod was mounted onto a pivotal axis so that the wooden ball would act as a pendulum to turn freely around the pivotal axis, as shown in Figure 3. The apple was cut in two equal parts in the direction of the stem-calyx axis before the impact test, to avoid any impact damage in the contact plane between the apple and the platform. The same parts that had been used for Vis/SWNIR spectra acquisition was selected for impact test. Based on preliminary test, three levels of impact energy $(1.11 \mathrm{~J}, 0.66 \mathrm{~J}$, and $0.33 \mathrm{~J})$ were chosen to create different bruise volumes for the test fruits. This was accomplished by rotating the wooden-ball pendulum upward at $\beta=90^{\circ}, 35^{\circ}$ or $15^{\circ}$, respectively, from the horizontal direction and then releasing the pendulum to allow it to fall freely until it hit the apple at the equatorial area. The samples used for different impact energy levels were of equal size (20 samples in each energy level in the first week and 40 samples in each level in the second and third weeks respectively). After the impact test, the samples were kept at room temperature for about $2 \mathrm{~h}$ until bruised tissue of the fruit flesh turned into brown.

A CCD camera (Fire-I; Unibrain Inc., San Ramon, Cal., USA) was used to obtain image of the bruised surface (Figure 4a). After acquiring bruised surface, the fruit was cut to two parts from the middle of the bruised spot perpendicularly to the fruit surface, and these two parts were rotated with $90^{\circ}$ and put together with the bruised depth profile outside as shown in Figure $4 \mathrm{~b}$. The image of bruised depth profile of two parts was also acquired for calculating the bruise volume.

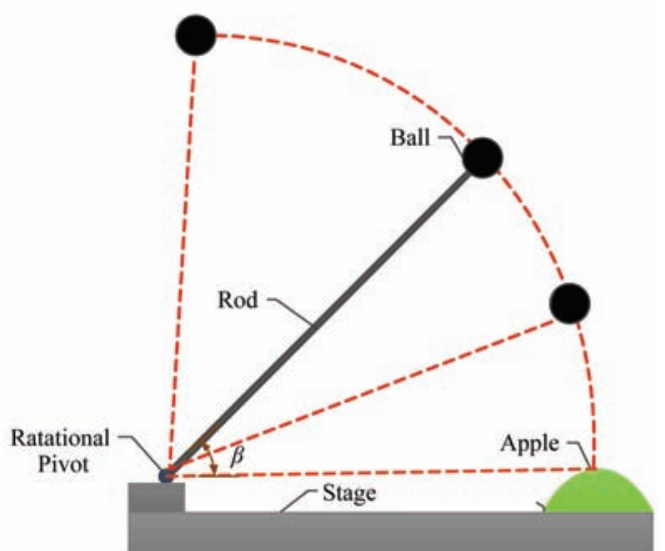

Figure 3 A wooden-ball pendulum impacting device for impact test on apples

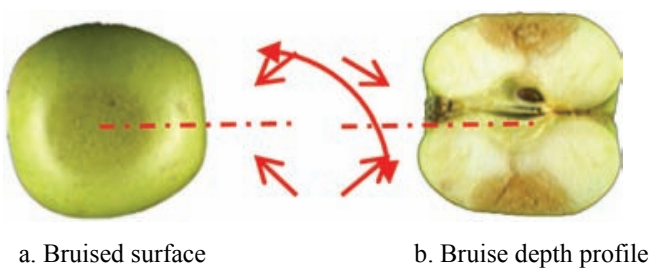

Figure 4 Bruised area of apple

The images of the bruised area (surface and depth profile) of the apples were segmented using Adobe PhotoShop CS6 (Adobe Systems Inc., San Jose, Cal., USA), and the contours of the bruised surface and bruise depth profile for one of two parts after segmentation were shown as black circular and semi-circular curves in Figures $5 \mathrm{a}$ and $5 \mathrm{~b}$, respectively. The bruise thickness method ${ }^{[19]}$ is often used to evaluate bruise volume in fruits due to its simplicity of calculation. The model considers the bruised area as a section of a sphere, and it is calculated for a spherical shape defined below the contact plane (point of maximum deflection of the apple during impact). The bruise volume $V_{C}\left(\mathrm{~mm}^{3}\right)$ was calculated as follows:

$$
V_{C}=\frac{\pi d}{24}\left(3 w^{2}+4 d^{2}\right)
$$

where, $w$ is the equivalent diameter of the bruise surface when it is approximated as a circle, $\mathrm{mm}$; $d$ is the equivalent depth of the bruised tissue, mm. As shown in Figure 5, the area of the enclosed black solid line is the actual bruise area. Assuming that the actual bruise surface area is $S_{1}\left(\mathrm{~mm}^{2}\right)$ and the area of the bruised depth profile is $S_{2}\left(\mathrm{~mm}^{2}\right)$, which were obtained by image segmentation. The circular and sectorial areas surrounded by red dotted lines are respectively equal to $S_{1}$ and $S_{2}$ based on the area equivalence principle; diameter 
$w$ and depth $d$ can be obtained by using Equations (3)-(6).

$$
\begin{gathered}
S_{1}=\frac{\pi}{4} w^{2} \\
\sin \frac{\theta}{2}=\frac{w}{2 R} \\
S_{2}=\frac{\theta}{2} R^{2}-\frac{\sin \theta}{2} R^{2} \\
d=R-\sqrt{R^{2}+\frac{w^{2}}{4}}
\end{gathered}
$$

where, $\theta$ and $R$ are the central angle (rad) and corresponding radius of the pie section $(\mathrm{mm})$, which can be calculated by the Equations (4) and (5). Finally, parameter $d$ is obtained by Equation (6). Then, the bruise volume $V_{c}$ is calculated by Equation (2) with the known parameters $w$ and $d$ (the average value of two parts). The impact energy $E$ can be calculated by Equation (7):

$$
E=\mathrm{m}_{b} g h+m_{r} g \frac{h}{2}
$$

where, $m_{b}$ and $m_{r}$ respectively represent the mass of the wooden ball and aluminum rod, $\mathrm{kg} ; h$ is the drop height of the wooden ball (m), which can be calculated from the drop angel $\beta$; and $g$ is the gravitational acceleration (9.8 $\mathrm{m} / \mathrm{s}^{2}$ ).

Finally, the bruise susceptibility in apple, $B S^{[8]}$ $\left(\mathrm{mm}^{3} / \mathrm{J}\right)$ can be calculated by Equation (8), where $E(\mathrm{~J})$ and $V\left(\mathrm{~mm}^{3}\right)$ respectively represent the impact energy and bruise volume.

$$
B S=\frac{V}{E}
$$

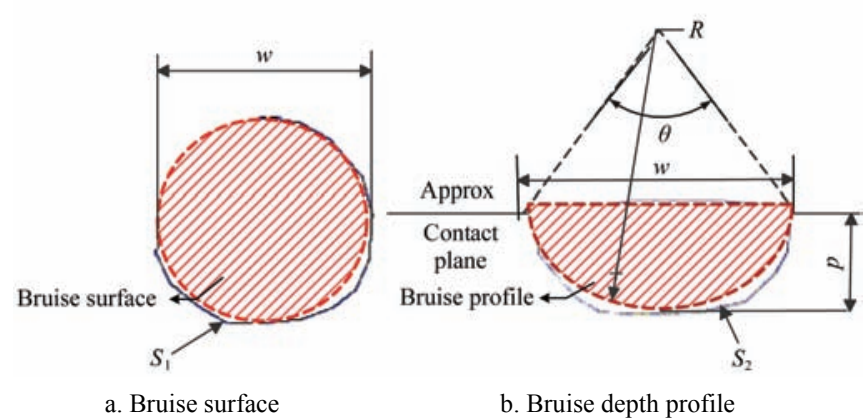

Figure 5 Method for evaluating the bruise volume

\subsection{Developing predicting model using selective} ensemble learning model based on feature selection (SELFS)

After obtaining spectra and bruise susceptibility of apples, predicting model which can reflect the relationship between the Vis/SWNIR spectra and the bruise susceptibility of apple need to be developed. To improve the generalization ability of the model, this work used the selective ensemble learning model based on feature selection (SELFS) to predict bruise susceptibility in apples. The SELFS mainly consists of individual generation, individual learner, and conclusion synthesis (denoted as I, II, and III in Figure 6). The individual generation is mainly used to generate a certain number of calibration subsets from whole calibration samples for developing individual learner. In this study, the calibration subsets were produced using feature selection method based on successful projection algorithm (SPA). The individual learner is mainly designed to establish a sub-model for each calibration subset. Partial least square (PLS) regression is a widely used chemometric method for building prediction model. PLS is suited well when the matrix of predictors has more variables than observations, and also when the input variables contain noise and are strongly correlated ${ }^{[20]}$. Therefore, the PLS was adopted in constructing the individual learner. The conclusion synthesis requires the integrated output of the results of every sub-model by a certain conclusion generation method. The SELFS method used in this work was summarized as follows:

1) Firstly, the SPA was used to select the important waveband subset from the full wavebands ${ }^{[21]}$, and $K$ additional wavebands were randomly selected from the residual wavebands, all of selected wavebands formed the final feature set, which were used as the input of the sub-model.

2) Secondly, the PLS sub-model for each selected wavebands was developed. The leave-one-out cross validation was used to determine the number of latent variables or factors, and the cross-validation correlation coefficient $r_{c v}$ of the training set and the prediction results of the training and prediction sets $\left(y_{c}\right.$ and $\left.y_{v}\right)$ were recorded. $L$ candidate sub-models were obtained by repeating steps 1 and 2 .

3) Finally, $L_{1}$ sub-models with a large value of $r_{c v}$, were selected from $L$ sub-models, and the final output was obtained by the weighted average. The specific formulas were expressed as Equations (9) and (10). 


$$
\begin{gathered}
Y_{c c}=\sum_{i=1}^{L_{1}} y_{c}^{i} \times \frac{r_{c v}^{i}}{\sum_{i=1}^{L_{1}} r_{c v}^{i}} \\
Y_{v v}=\sum_{i=1}^{L_{1}} y_{v}^{i} \times \frac{r_{c v}^{i}}{\sum_{i=1}^{L_{1}} r_{c v}^{i}}
\end{gathered}
$$

where, $r_{c v}^{i}$ represents the cross-validation correlation coefficient of the $i^{t h}$ selected sub-model; and $Y_{c c}, Y_{v v}$ represent the integrated output of the training and prediction sets, respectively.

The number $K$ of randomly selected wavebands, the number $L$ of candidate sub-models, and the final number $L_{1}$ of individual learners have impacts on the final model performance. A considerably small $K$ value may weaken the learning performance of the individual learner. By contrast, a considerably large $K$ value may reduce the number of differences between individual learners in terms of learning performance, eliminating the complementary superiorities between individual learners in ensemble learning ${ }^{[22,23]}$. A large $L$ value of the candidate individual learners is conducive for selecting highly complementary individual learners; however, an excessively large $L$ value of the individual learners may bring computational complexity to the model. With a considerably low $L_{1}$ value of the individual learners, the complementary strengths of the individual learners is difficult to use in learning performance, whereas a considerably large $L_{1}$ value of the individual learners are likely to result in poor performance of the sub-models, weakening the final output performance. Considering the above factors and combining the priori experiments, the number $K$ of the randomly selected wavebands is set at $1 / 10$ of the number of the residual wavebands, the number $L$ of the candidate individual learners is set at $L=20$, and the final number $L_{1}$ of individual learners is set at $L_{1}=10$.

\subsection{Evaluation of model performance}

In this study, four models were developed based on three levels of impact energy and their pooled data. Prior to the model development, $3 / 4$ of samples (75 samples for the three levels of impact energy and 225 for their pooled data) were randomly selected and used as calibration set, and the remaining $1 / 4$ samples (25 samples for the three levels of impact energy and 75 samples for their pooled data) were used as prediction set. The correlation coefficient of the calibration $\left(R_{c}\right)$ and prediction $\left(R_{p}\right)$, root-mean-square error of the calibration (RMSEC) and prediction (RMSEP), and the residual predictive deviation $(R P D)$ which is the ratio of the sample standard deviation to RMSEP were the five indicators used to evaluate the prediction ability of the model. Given the impacts of the calibration set on the performance of the model, the sample selection method for calibrations and predictions was repeated 10 times, and the mean values of $R_{c}, R_{p}, R M S E C, R M S E P$ for ten runs were calculated for evaluating model performance. All data were analyzed using Matlab software (R2009b, Mathorks, Inc., Natick, Mass., USA).

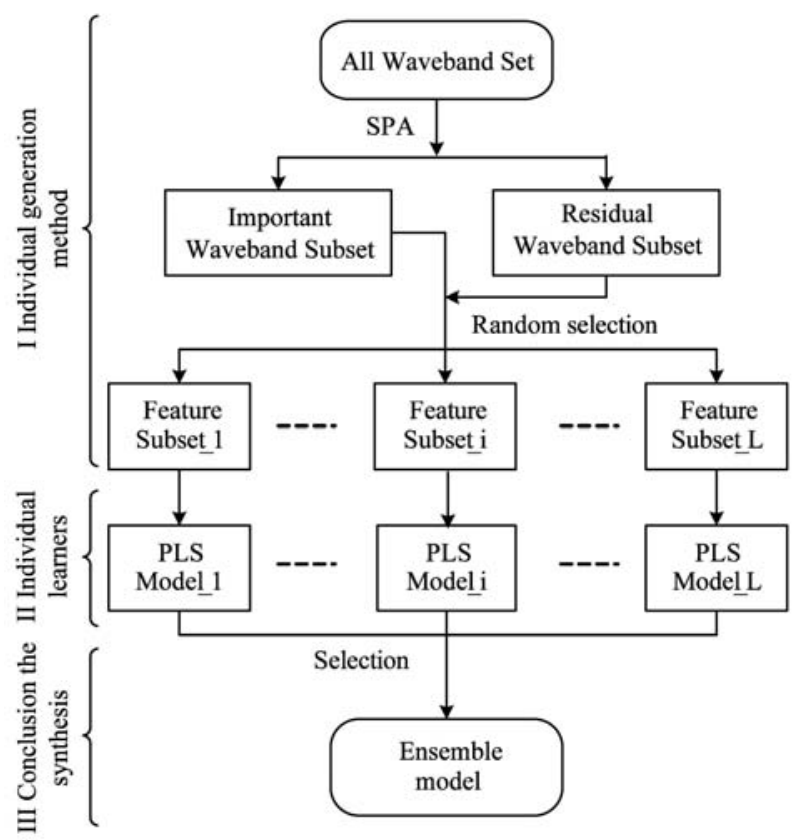

Figure 6 Flowchart of the selective ensemble learning model based on feature selection

\section{Results and discussion}

\subsection{Analysis of bruise susceptibility}

Table 1 shows the statistical results on bruise susceptibility and bruise surface area of 300 'Golden delicious' apples. The mean value for the bruise susceptibility $(B S)$ in apple increased with the decrease of impact energy, ranging from $455.3 \mathrm{~mm}^{3} / \mathrm{J}$ for 'High Impact' at $1.11 \mathrm{~J}$ to $646.4 \mathrm{~mm}^{3} / \mathrm{J}$ for 'Low Impact' at $0.33 \mathrm{~J}$ (Table 1). The $t$-test for the bruise susceptibility shown that there were significant differences among bruise susceptibilities at three levels of impact energy, 
with $p$-value of $8.36 \times 10^{-28}, 3.36 \times 10^{-31}$, and $1.97 \times 10^{-4}$ for the bruise susceptibility at high and medium, high and low, and medium and low, respectively. This implied that apples were more susceptible to bruising at lower impact than at high impact. This may be due to the fact that the contact area between the apple and wooden ball increased with the increase of impact energy. Larger contact area would be more efficient in dispersing the impact energy in the fruit from the impacting wooden ball and the impact energy per unit area would be reduced, which would lead to a decrease in plastic deformation and reduce the possibility in further tissue structure damage $^{[24]}$. This finding is agreement with the results of Marashall ${ }^{[25]}$, which reported that the ratio of bruise volume of 'Golden Delicious' apples to energy absorbed decreased with the increase of the energy absorbed.

Table 1 Statistics for the susceptibility and bruise surface area of 300 'Golden Delicious' apples at three levels of impact energy

\begin{tabular}{|c|c|c|c|c|c|c|c|c|c|}
\hline \multirow{2}{*}{ Impact Level } & \multirow{2}{*}{ Number } & \multicolumn{4}{|c|}{ Bruise Susceptibility $/ \mathrm{mm}^{3} \cdot \mathrm{J}^{-1}$} & \multicolumn{4}{|c|}{ Bruise surface area $/ \mathrm{mm}^{2}$} \\
\hline & & Maximum & Minimum. & Mean & SD & Maximum & Minimum. & Mean & SD \\
\hline $\operatorname{High}(E=1.11 \mathrm{~J})$ & 100 & 581.7 & 353.3 & 455.3 & 60.7 & 164.6 & 137.4 & 151.1 & 5.9 \\
\hline $\operatorname{Medium}(E=0.66 \mathrm{~J})$ & 100 & 748.9 & 419.4 & 600.4 & 92.0 & 144.4 & 113.0 & 126.5 & 7.3 \\
\hline Low $(E=0.33 \mathrm{~J})$ & 100 & 880.5 & 444.5 & 646.4 & 113.9 & 108.3 & 79.5 & 92.7 & 6.4 \\
\hline Pooled & 300 & 880.5 & 353.3 & 567.3 & 122.4 & 164.6 & 79.5 & 124.5 & 23.9 \\
\hline
\end{tabular}

\subsection{Prediction results for apple bruise susceptibility}

The PLS model based on whole spectral wavebands (namely PLS), the PLS model based on optimal wavebands selected by SPA (namely SPA-PLS), and the SELFS model were developed and compared in this study. Table 2 shows the calibration and prediction results for apple bruise susceptibility for three levels of impact energy (100 samples for each level) and their pooled data (300 samples). Overall, a significance test $(p \leq 0.05)$ for the RMSEP shown there were no significant differences between PLS model and SPA-PLS model. The reason may contributed that SPA-PLS only uses a small amount of important wavebands to develop calibration model, which may bring the risk of useful wavebands loss, and ultimately lead to the weakening of the SPA-PLS model performance. Compared with PLS and SPA-PLS model, the SELFS model obtained the best performance, with the $R_{p}$ values of $0.830,0.887,0.867$ and 0.731 for high, medium, low energy, and pooled data, respectively. The RMSEP values obtained by SELFS models decreased by $20.0 \%$ and $21.2 \%$ for high energy, $17.6 \%$ and $18.6 \%$ for medium energy, $14.8 \%$ and $11.4 \%$ for low energy, $3.4 \%$ and $3.7 \%$ for pooled data, compared with the PLS models and SPA-PLS models, respectively. The SELFS models achieved the $R P D$ values of 1.78 for high energy, 2.14 for medium energy, 1.94 for low energy, and 1.46 for pooled data, which improved by $24.5 \%, 20.2 \%, 15.0 \%$ and $3.4 \%$ over the PLS models, and 27.1\%, 22.3\%, 11.5\% and $3.4 \%$ over the SPA-PLS models, for three impact energy and their pooled data. A significance test $(p \leq 0.05)$ for the RMSEP and the RPD shown that the SELFS models were significantly superior to PLS models and SPA-PLS models for each impact energy level as well as for the pooled data. All of prediction model had a lower value of $R_{p}(<0.75)$ and $R P D(<1.46)$ for the pooled data. In this study, the impact energy was considered to be completely absorbed by apple, while the energy loss resulting from the rebound of the impacting ball was ignored. This approximation could have introduced errors for the pooled data because the rebound height for each impact energy level was different, which might have contributed to a lower $R_{p}$ value and $R P D$ for the pooled data. The RPD between 1.5 and 2 means that the model can discriminate low from high values of the response variable. Although SEFLS model didn't yield the exceptionally high RPD values for the pooled data in this study, it was still meaningful for estimating bruise susceptibility. Figure 7 shows the prediction results for bruise susceptibility versus the actual measurements for one of ten runs for the high, medium and low energy as well as for the pooled data, respectively, using SELFS. Overall, there were good, but not exceptional, correlations between Vis/SWNIR spectra and bruise susceptibility. 
Table 2 Results of PLS, SPA-PLS and SELFS models for the bruise susceptibility of 'Golden Delicious' apples *

\begin{tabular}{|c|c|c|c|c|c|c|}
\hline Impact Level & Model & $R_{c}$ & $R_{p}$ & $\begin{array}{c}\text { RMSEC/ } \\
\mathrm{mm}^{3} \cdot \mathrm{J}^{-1}\end{array}$ & $\begin{array}{c}R M S E P / \\
\mathrm{mm}^{3} \cdot \mathrm{J}^{-1}\end{array}$ & $R P D$ \\
\hline \multirow{2}{*}{$\begin{array}{c}\text { High } \\
(E=1.11 \mathrm{~J})\end{array}$} & PLS & 0.839 & 0.753 & 32.24 & 43.30 & 1.43 \\
\hline & SELFS & 0.878 & 0.830 & 29.15 & 34.74 & 1.78 \\
\hline \multirow{3}{*}{$\begin{array}{l}\text { Medium } \\
(E=0.66 \mathrm{~J})\end{array}$} & PLS & 0.959 & 0.842 & 23.68 & 53.93 & 1.78 \\
\hline & SPA-PLS & 0.961 & 0.839 & 22.77 & 54.64 & 1.75 \\
\hline & SELFS & 0.940 & 0.887 & 30.9 & 44.46 & 2.14 \\
\hline \multirow{3}{*}{$\begin{array}{c}\text { Low } \\
(E=0.33 \mathrm{~J})\end{array}$} & PLS & 0.957 & 0.829 & 29.74 & 73.05 & 1.67 \\
\hline & SPA-PLS & 0.946 & 0.836 & 35.12 & 70.22 & 1.74 \\
\hline & SELFS & 0.919 & 0.867 & 43.79 & 62.22 & 1.94 \\
\hline \multirow[b]{2}{*}{ Pooled } & PLS & 0.770 & 0.706 & 77.42 & 88.48 & 1.41 \\
\hline & SPA-PLS & 0.769 & 0.704 & 77.59 & 88.71 & 1.41 \\
\hline
\end{tabular}

Note: $* R_{c}$ and $R_{p}=$ correlation coefficient for the calibration set and the prediction set, respectively, $R M S E C$ and $R M S E P=$ root-mean-square error of the calibration set and the prediction set, respectively, SELFS = selective ensemble learning model based on feature selection, and $R P D=$ ratio of sample standard deviationt to $R M S E P$.
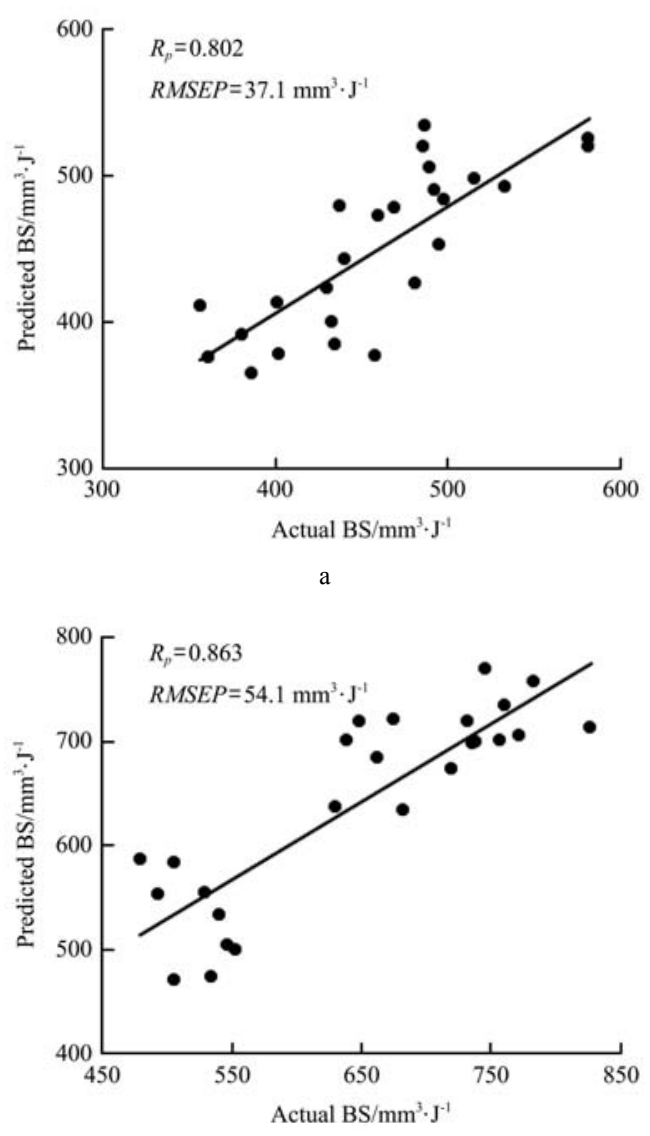

$\mathrm{c}$

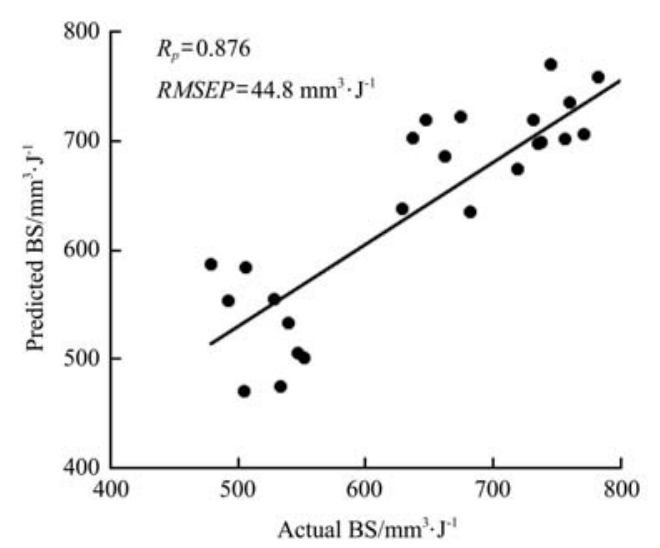

b



d

Figure 7 Prediction results from one of the ten model runs for the bruise susceptibility in apples using SELFS models at (a) high ( $E=1.11 \mathrm{~J}$ ), (b) medium ( $E=0.66 \mathrm{~J})$, (c) low $(E=0.33 \mathrm{~J})$, impact energy levels, and (d) pooled data of three impact energy levels

\subsection{Discussion}

Accurate prediction of bruise susceptibility can help design appropriate fruit handling systems to reduce or control bruising damage to apples during harvest and postharvest handling. In this research, application of Vis/SWNIR for fruit bruise susceptibility prediction is based on the premise that the fruit bruise susceptibility is affected by physiological and structural characteristics of fruits, and difference in physiology and structure of fruit would lead to different reflectance spectra. Considering the redundant information among all spectral wavebands, SELFS was used to establish a prediction model to make full use of the spectral information, and to improve the model prediction accuracy and robustness. The results 
clearly demonstrated that the bruise susceptibility of apples is related to the tissue optical property, and it could be predicted by Vis/SWNIR spectroscopy. The results also proved that the SELFS was an effective modeling method for improving prediction accuracy for bruise susceptibility, compared with PLS model and SPA-PLS model. The Vis/SWNIR combined with SELFS obtained good, but exceptionally high accuracy, as indicted by $R_{p}$ and $R P D$. This may be contributed the flowing three factors. First, the bruise susceptibility is directly related to the structural and mechanical properties of tissue, which is mainly reflected in the scattering properties of tissue. $3.5 \mathrm{~mm}$ separating distance between the light illuminating ring and the detector used in this experiment may not be able to collect enough information about the scattering property due to the short scattering distance. Second, the energy loss resulting from the rebound of the impacting ball was ignored in this study, which introduced errors for calculating $B S$ value of apples because the rebound height for each apple was different. Finlay, errors were also expected in estimating the bruise volume of apples by the image segmentation technique and the volume estimation model, which could have a negative effect on the BS prediction of apples. Zhu et al. ${ }^{[25]}$ developed the PLS model for bruise susceptibility prediction of 300 'Golden Delicious' apples, which is same with this study, using hyperspectral scattering imaging technique. They reported the better prediction results, with $R_{p}$ values of $0.85,0.92$ and 0.90 for high, medium and low energy as well as 0.83 for the pooled data, compared with this study. The better prediction results obtained by hyperspectral image technique attributed to fact that hyperspectral imaging is based on spatially-resolved technique, thus, the spectra calculated from the scattering profile contain more information about the samples. Compared with hyperspectral scattering imaging, Vis/SWNIR spectroscopy has the advantage of low-price and fast detection speed, it has been commercially used for online detection of fruit quality. The method proposed in this study can be easily integrated into the existing commercial systems, and realize the simultaneous measurement of multiple parameters (i.e. SSC, BS), without increasing the cost of existing equipment. Hence, the Vis/SWNIR is a potential tool in online evaluation for bruise susceptibility of fruits.

\section{Conclusions}

In this study, spectroscopy covering 400-1100 nm was used to assess bruise susceptibility of fresh 'Golden Delicious' apples. To improve the generalization ability and robustness of models, the SELFS method was adopted for developing prediction model of bruise susceptibility of apples. Compared with the PLS model and SPA-PLS model, the SELFS model demonstrates better prediction results at the three energy levels, with $R_{p}$ for prediction set higher than 0.800 and an RMSEP of lower than $62.1 \mathrm{~mm}^{3} / \mathrm{J}$; moreover, in predicting bruise susceptibilities of pooled data, $R_{p}$ of 0.731 and $R M S E P$ of $85.46 \mathrm{~mm}^{3} / \mathrm{J}$ was obtained. When SELFS was used for developing BS prediction model, it improved RMSP value over the PLS models by $14.8 \%-20.0 \%$ for three impact energies and $3.4 \%$ for their pooled data, and the SPA-PLS models by $11.4 \%-21.2 \%$ for three impact energies and $3.4 \%$ for their pooled data, respectively. The SELFS models achieved the $R P D$ values of 1.78-2.14 for three impact energies and 1.46 for pooled data, with improvements of $15.0 \%-24.5 \%$ for three impact energies and $3.4 \%$ for pooled data over the PLS models, and $11.5 \%-27.1 \%$ for three impact energies and $3.4 \%$ for pooled data over the SPA-PLS, respectively. The results demonstrated that Vis/SWNIR combined with SELFS could provide a new, cost-effective tool for nondestructive evaluation of bruise susceptibility for individual fruit. The technique could be useful in fruit sorting and packaging. However, further research is needed on improving spectral measurement of spectroscopy and for more varieties of apple under different maturity and postharvest storage conditions, so that more accurate prediction of bruise susceptibility can be achieved.

\section{Acknowledgements}

The authors would like to thanks Renfu $\mathrm{Lu}$ and Fernando Mendoza in the USDA Agricultural Research Service (ARS) Sugarbeet and Bean Research Unit at East 
Lansing, Michgan, for their technical support for the experiment. The research was also supported by the Chinese National Natural Science Foundation (61275155, 61271384).

\section{[References]}

[1] Opara L U. Bruise susceptibilities of 'Gala' apples as affected by orchard management practices and harvest date. Postharvest Biology and Technology, 2007; 43(1): 47-54.

[2] Umezuruike L O, Pankaj B P. Bruise damage measurement and analysis of fresh horticultural produce: A review. Postharvest Biology and Technology, 2014; 91: 9-24.

[3] Lu R. Detection of bruises on apples using near-infrared hyperspectral imaging. Transaction of ASAE, 2003; 46: 523-530.

[4] Bajema R W, Hyde G M. Instrumented pendulum for impact characterization of whole fruit and vegetable specimens. Transactions of the ASAE, 1998; 41: 1399-1405.

[5] Baritelle A L, Hyde G M. Commodity conditioning to reduce impact bruising. Postharvest Biology and Technology, 2001; 21: 331 - 339.

[6] Van Linden V, Scheerlinck N, Desmet M, De Baerdemaeker J. Factors that affect tomato bruise development as a result of mechanical impact. Postharvest Biology and Technology, 2006; 42(3): 260-270.

[7] Pang D W, Studman C J, Banks N H, Baas P H. Rapid assessment of the susceptibility of apples to bruising. Journal of Agricultural Engineering Research, 1996; 64(1): 37-47.

[8] Bajema R W, Hyde G M. Instrumented pendulum for impact characterization of whole fruit and vegetable specimens. Transactions of the ASAE, 1998; 41(5): 1399 1405.

[9] Abedi G, Ahnadi E. Bruise susceptibilities of Golden Delicious apples as affected by mechanical impact and fruit properties. The Journal of Agricultural Science, 2013; 152(3): 439-447.

[10] Opara L U. Bruise susceptibilities of 'Gala' apples as affected by orchard management practices and harvest date. Postharvest Biology and Technology, 2007; 34(1): 47-54.

[11] Khan A A, Vincent J F V. Anisotropy in the fracture properties of apple flesh as investigated by crack-opening tests. Journal of Materials Science, 1993; 28(1): 45-51

[12] Khan A A, Vincent J F V. Compressive stiffness and fracture properties of apple and potato parenchyma. Journal of Texture Studies, 1993; 24(4): 423-435.

[13] Gacia J, Ruiz-Altisent M, Barreiro P. Factors influencing mechanical properties and bruise susceptibility of apples and pears. Journal of Agricultural Engineering Research, 1995; 61(1): 11-17.

[14] Schoorl D, Holt J E. Bruise resistance measurements in apples. Journal of Texture Studies, 1980; 11(4): 389-394.

[15] Opara L U, Al Ghafri A, Agzoun H, Al Issai J, Al Jabri F. Design and development of a new device for measuring susceptibility to impact damage of fresh produce. New Zealand Journal of Crop and Horticultural Science, 2007; 35(2): 245-251.

[16] Pan L, Zhu Q, Lu R, Mitchell McGrathd J. Determination of sucrose content in sugar beet by portable visible and near-infrared spectroscopy. Food Chemistry, 2015; 167: 264-271.

[17] Subedi P, Walsh K. Assessment of sugar and starch in intact and mango fruit by SWNIR spectroscopy. Postharvest Biology and Technology, 2011; 62(3): 41-47.

[18] Wu G, Wang C. Investigating the effects of simulated transport vibration on tomato tissue damage based on Vis/NIR spectroscopy. Postharvest Biology and Technology, 2014; 98: 41-47

[19] Bollen A F, Nguyen H X, Dela Rue B T. Comparison of methods for estimating the bruise volume of apples. Journal of Agricultural Engineering Research, 1999; 74(4): 325-330.

[20] Mendoza F, Lu R, Cen H. Comparison and fusion of four nondestructive sensors for predicting apple fruit firmness and soluble solids content. Postharvest Biology and Technology, 2012; 73: 89-98

[21] Araujo M C U, Saldanha T C B, Galvao R K H, Yoneyama T, Chame H C, Visani V. The successive projections algorithm for variable selection in spectroscopic multicomponent analysis. Chemometrics and Intelligent Laboratory Systems, 2001; 57(2): 65-73.

[22] Zhou Z, Wu J, Tang W. Ensembling neural networks: Many could be better than all. Artificial Intelligence, 2002; 137(1): 239-263.

[23] Perrone M P. When networks disagree: Ensemble method for neural networks. London: U. S. Army Research Office, 1992.

[24] Gołacki K, Bobin G, Stropek Z. The determination of apples bruise resistance by the multiple impact method. Technology Science, 2009; 12: 40-47.

[25] Marshall D E, Burgess G J. Apple bruise damage estimation using an instrumented sphere. Applied Engineering in Agriculture, 1991; 6: 677-682.

[26] Zhu Q, Guan J, Huang M, Lu R, Mendoza F. Predicting bruise susceptibility of Golden Delicious' apples using hyperspectral scattering technique. Postharvest Biology and Technology, 2016; 114: 86-94. 